\title{
Response to rapamycin analogs but not PD-I inhibitors in PTEN-mutated metastatic non-small-cell lung cancer with high tumor mutational burden
}

This article was published in the following Dove Press journal:

Lung Cancer:Targets and Therapy

\section{Ankur R Parikh' \\ Siraj MAli² \\ Alexa B Schrock ${ }^{2}$ \\ Lee A Albacker ${ }^{2}$ \\ Vincent A Miller ${ }^{2}$ \\ Phil J Stephens ${ }^{2}$ \\ Pamela Crilley' \\ Maurie Markman' \\ 'Eastern Regional Medical Center, \\ Cancer Treatment Centers of America, Philadelphia, PA, USA; \\ ${ }^{2}$ Foundation Medicine, Inc, Cambridge, MA, USA}

Video abstract

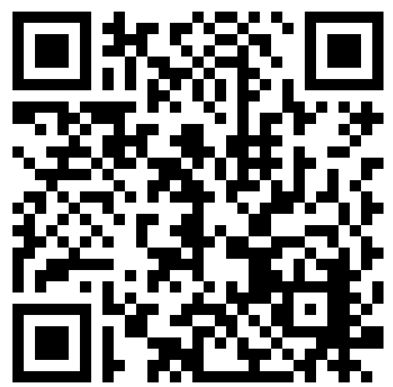

Point your SmartPhone at the code above. If you have a QR code reader the video abstract will appear. Or use: http://youtu.be/5RIYKhxO_Us

Correspondence: Ankur R Parikh

Eastern Regional Medical Center, Cancer

Treatment Centers of America, I33 I

E. Wyoming Avenue, Philadelphia, PA

19124, USA

Tel +I 2I55377400

Email ankur.parikh@ctca-hope.com

\begin{abstract}
In non-small-cell lung cancer (NSCLC) refractory to standard therapy and which lacks well-known oncogenic drivers, genomic profiling can still identify genomic alterations that may suggest potential sensitivity to targeted therapy. PTEN mutation in NSCLC may be sensitizing to analogs of rapamycin such as everolimus or temsirolimus, but more investigation is needed. We report the case of a patient with metastatic NSCLC harboring a PTEN mutation as well as high tumor mutational burden and PD-L1 positivity with a durable response to temsirolimus, but refractory to a checkpoint inhibitor. Even in the event of failure of treatment with checkpoint inhibitors in the background of a case with a higher tumor mutational burden and PD-L1 positivity, targeting specific genomic alterations may still result in patient benefit. Keywords: genomic profiling, temsirolimus, targeted therapy, immunotherapy
\end{abstract}

\section{Introduction}

Lung cancer is one of the most common cancers diagnosed in the US. The 5-year survival upon diagnosis of stage IV lung cancer is $4 \% .{ }^{1}$ Comprehensive genomic profiling is being used more frequently to help identify mutations in an individual patient's non-small-cell lung cancer (NSCLC) to suggest benefit from targeted therapy or immunotherapy. We present the case of a patient with metastatic NSCLC found to harbor PTEN and STK11 alterations using comprehensive genomic profiling who responded to matched targeted therapy in the background of higher tumor mutational burden.

\section{Case history}

The patient is a 62-year-old woman with a 60 pack-year smoking history who presented with stage IV lung adenocarcinoma in September 2012. She was found to have bilateral disease in her lungs, a right adrenal mass, and osseous metastasis. EGFR and $A L K$ were queried by single gene molecular testing. The patient enrolled in a clinical trial and was treated with carboplatin, paclitaxel, and bevacizumab. She responded to therapy and was then placed on maintenance bevacizumab. She had disease progression in February 2013 and was treated with pemetrexed. Her disease progressed in November 2013, and so she was treated with docetaxel. Her computed tomography scans showed response at 3 months and 6 months; however, by 9 months, she had further disease progression including a new intracranial metastasis. She received stereotactic 
radiation to her intracranial metastasis in May 2014. She was then started on gemcitabine and found to have disease progression after 3 cycles.

To identify opportunities for possible benefit from targeted therapy, comprehensive genomic profiling (FoundationOne, Foundation Medicine, Inc., Cambridge, MA, USA) was performed on a biopsy of a right upper lobe lesion collected in December 2013, which revealed the following genomic alterations: PTEN D268fs*30, STK11 splice site 465-1G>T, p53 G293R, KEAP1 G480W, and $L R P 1 B$ G528W, as well as tumor mutational burden of 18.9 mutations/megabase. On the basis of PTEN and STK11 alterations, the decision was made to treat the patient with weekly temsirolimus $25 \mathrm{mg}$ IV weekly in analogy to dosing for advanced renal cell carcinoma. After 3 months of treatment, she showed significant clinical improvement in her breathing and overall functional status. Although her radiologic response was not formally evaluated by RECIST criteria, it was analogous to a robust partial response with significant reduction of a right lung mass and reduction of a pleural mass (Figure 1). She remained on treatment for almost 20 months until March 2016 when her scans showed disease progression with increase in size of right-sided lung mass as well as increase in pleural effusion. Her treatment was then changed to nivolumab, on which the disease progressed after 3 months. Treatment was changed to vinorelbine in July 2016 with partial response, but by January 2017, she had clinical and radiographic progression. Pleural fluid drained in January 2017 showed 80\% PD-L1 expression
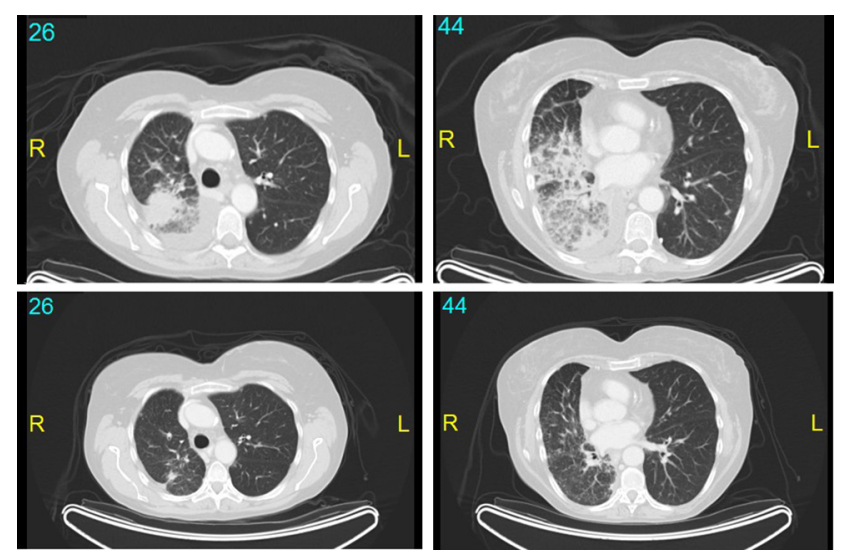

Figure I The top two images are from July 2014, and bottom two images are from October 2014 after 3 months of treatment with temsirolimus.

Notes: There was a significant decrease in the size of the right upper lobe mass which abuts the major fissure. There was also marked improvement in the airspace consolidation involving the right lower lobe. Minimal amount of interstitial thickening remained within the right lower lobe. There was significant improvement in right pleural disease. There is no significant pleural effusion. A $1 \mathrm{~cm}$ right upper lobe pulmonary nodule had decreased in size. No significant mediastinal, axillary, or hilar lymphadenopathy was observed. No pericardial effusion was seen. Tiny nodules previously noted in the left lower lobe decreased in size. by immunohistochemistry. She was then treated with pembrolizumab in February 2017, but after 2 cycles had further clinical and radiographic progression. The patient transferred to a hospice in March 2017 and passed away a month later.

\section{Discussion}

The exceptional response to a rapamycin analog in this patient and the underlying mechanism likely hinges on the mutations of PTEN and possibly, to a lesser extent, on STK11, which are both regulators of the downstream mTOR pathway, and the former is known to confer sensitivity to rapamycin analogs in vivo. Given that $P T E N$ is a tumor suppressor, loss of heterozygosity $(\mathrm{LOH})$ was queried on a research basis for both loci, but due to low sample purity could not be determined for either PTEN or STK11 (data not shown).

Loss of function of PTEN, a lipid phosphatase, has long posited as being sensitizing to rapamycin due to ensuing hyperactivation of mTORC $1 .^{2}$ In particular, biallelic loss of function of PTEN should be more sensitizing than mutation of a single allele. ${ }^{2}$

STK11 is also upstream of mTOR, and loss of function alterations of STK11 also hyperactivate mTORC1, albeit through the intermediary of AMPK activity. However, in preclinical models of STK11-driven dysplasia and neoplasia, only some of these entities are rapamycin sensitive, such the polyps of juvenile polyposis and observation in PIN. ${ }^{3}$ Overall, whether STK11 alteration confers sensitivity to rapamycin analogs remains poorly understood.

A significant limitation in this study is the inability to identify whether PTEN is under $\mathrm{LOH}$, which would offer a compelling explanation for the efficacy of temsirolimus. The specimen purity did not permit LOH assessment, and no additional specimens were available including original sample (data not shown). Future investigation, whether on the basis of an exceptional responder to rapamycin analog like the patient here or in the context of clinical investigation where the therapeutic hypothesis is a similar question, should incorporate genomic assessment of both alleles of PTEN to help further explain the mechanism of the effect seen here. Studies of both temsirolimus and everolimus in unselected prostate cancer populations did not show an effect, but the latter trended toward some efficacy in a population with PTEN alteration. ${ }^{4,5}$

One aspect of note is the relatively high tumor mutational burden observed with high PD-L1 staining, but lack of durable response to checkpoint inhibitor. This failure increases the impact of observing a prolonged response to genomically matched targeted therapy for this patient. 
In conclusion, the exceptional response observed here suggests that further assessment and investigation is warranted for PTEN status in NSCLC lacking known oncogenic drivers.

The authors apologize to all the investigators whose work cannot be cited here due to limitations of format. Written informed consent was provided by the patient to have the case details published.

\section{Disclosure}

ABS, LAA, VAM, PJS, and SMA are employees of and have equity interest in Foundation Medicine Inc. The authors report no other conflicts of interest in this work.

\section{References}

1. Siegel RL, Miller KD, Jemal A. Cancer statistics, 2017. CA Cancer J Clin. 2017;67(1):7-30.

2. Neshat MS, Mellinghoff IK, Tran C, et al. Enhanced sensitivity of PTENdeficient tumors to inhibition of FRAP/mTOR. Proc Natl Acad Sci USA. 2001;98(18):10314-10319.

3. Zhou W, Marcus AI, Vertino PM. Dysregulation of mTOR activity through LKB1 inactivation. Chin J Cancer. 2013;32(8):427-433.

4. Kruczek K, Ratterman M, Tolzien K, Sulo S, Lestingi TM, Nabhan C. A phase II study evaluating the toxicity and efficacy of single-agent temsirolimus in chemotherapy-naïve castration-resistant prostate cancer. Br J Cancer. 2013;109(7):1711-1716.

5. Templeton AJ, Dutoit V, Cathomas R, et al. Phase 2 trial of single-agent everolimus in chemotherapy-naive patients with castration-resistant prostate cancer (SAKK 08/08). Eur Urol. 2013;64(1):150-158.
Lung Cancer: Targets and Therapy

\section{Publish your work in this journal}

Lung Cancer: Targets and Therapy is an international, peer-reviewed, open access journal focusing on lung cancer research, identification of therapeutic targets and the optimal use of preventative and integrated treatment interventions to achieve improved outcomes, enhanced survival and quality of life for the cancer patient. Specific topics covered in the journal include: Epidemiology, detection and screening Cellular research and biomarkers; Identification of biotargets and agents with novel

Submit your manuscript here: https://www.dovepress.com/lung-cancer-targets--therapy-journa
Dovepress

mechanisms of action; Optimal clinical use of existing anticancer agents, including combination therapies; Radiation and surgery; Palliative care; Patient adherence, quality of life, satisfaction; Health economic evaluations. The manuscript management system is completely online and includes a very quick and fair peer-review system. Visit http://www.dovepress.com/testimonials.php to read real quotes from published authors. 